seizures recurred, even during hospitalization. Metabolic studies were considered. At one hospital stay, the seizure was complicated by ventricular tachycardia and fibrillation. After transfer to intensive-care for resuscitation, she was comatose and hypotonic, her pupils were dilated but reactive, and she was ventilated mechanically. She rapidly recovered consciousness, breathed independently, and moved all limbs. Mother was unimpressed and denied improvement in her child's condition. Toxicological studies obtained because of mother's incongruous response disclosed serum concentrations of amitryptyline $350 \mathrm{mcg} / \mathrm{L}$. Mother admitted that she had administered amitryptyline drops to the infant for the past month. When removed to live with maternal grandmother, the child remained well and without seizures. The child was a victim of the factitious disorder, Munchausen's syndrome by proxy (Astuto M et al. Lancet Jan 3, 2009;373:94).

\title{
MRI FINDINGS AND SEIZURE OUTCOMES COMPARED IN FOCAL CORTICAL DYSPLASIAS TYPES I AND II
}

Preoperative clinical, neuropsychological, EEG, and imaging features and surgical outcomes of focal cortical dysplasias (FCD) types I and II were compared in 40 children with refractory epilepsy and histopathologically confirmed FCD subtypes, in a study at Charles University, Prague, Czech Republic and centers in Germany. FCD type I was identified in 24 and FCD type II in 16 patients. Seizure types were secondarily generalized tonic-clonic in $54 \%$ of FCDI and 37\% FCDII; and infantile spasms in $21 \%$ of FCDI and $12 \%$ FCDII patients. Status epilepticus occurred in $4 \%$ of FCDI and $12 \%$ of FCDII patients. Multilobar involvement was found in $92 \%$ patients with FCDI and in $50 \%$ of FCDII patients. FCD type I MRI findings include white matter signal changes on T2 images and/or FLAIR, and reduction of white matter volume. FCD type II MRI showed increased cortical thickness, transmantle sign, gray-white matter junction blurring, fluid-attenuated inversion recovery (FLAIR) and proton density (PD) gray and white matter signal changes. Hippocampal atrophy occurred in only 1 patient of each type. Patients with FCD type I showed EEG continuous slowing more commonly; they had lower IQ levels and more behavioral disorders. Postsurgical seizure freedom occurred in 21\% FCD type I and in 75\% FCD type II patients $(\mathrm{P}<0.001)$. (Krsek $\mathrm{P}$, Pieper $\mathrm{T}$, Karimeier $\mathrm{A}$ et al. Different presurgical characteristics and seizure outcomes in children with focal cortical dysplasia type I or II. Epilepsia Jan 2009;50:125-137). (Respond: Pavel Krsek MD PhD, Department of Pediatric Neurology, Charles University, Second Medical School, Motol University Hospital, V Uvalu 84, CZ 15006 Prague 5, Czech Republic. E-mail:pavel.krsek@post.cz).

COMMENT. FCD is reported in up to $25 \%$ of surgical patients with intractable epilepsy and 5-10\% of epilepsies. FCD Ia and Ib, and FCD IIa and IIb are classified histopathologically. Type IIa is distinguished by dysmorphic, and IIb by balloon cells. FCD type I patients present within the first year of life, and have multilobar involvement, high seizure frequency, more complications, and a worse prognosis than FCD type II patients. FCDI is more frequently associated with mental retardation and behavioral disorders, more difficult to localize by EEG and MRI, and has less favorable surgical outcome. The authors consider surgery except for hemispherectomy is frequently palliative in FCDI cases. FCD type I should be considered a distinct entity. 